\title{
Mosel: A Sound and Efficient Tool for M2L(Str)
}

Peter Kelb, Tiziana Margaria, Michael Mendler, Claudia Gsottberger

Dept. of Comp. Science, Universität Passau, Innstr. 33, D-94032 Passau (Germany)

$\{k e l b, t i z i a n a$, mendler, gsottber $\}$ @mi . uni-passau.de

\section{Introduction}

Mosel [6] is a new system for the analysis and verification in Monadic SecondOrder Logic (M2L) based on model construction. The aim is to offer a systemlevel environment supporting several theories of the logic. MOSEL is a toolset, which, in its complete realization, will include a flexible set of decision procedures for several theories of the logic (e.g., finite and infinite strings, and trees) complemented by a variety of support components to provide input format translations, visualization, and interfaces to other logics and other analysis, verification, and synthesis tools. The presentation will concern the important concepts from the toolbuilders' perspective: design principles, implementation, efficiency and application domains.

\section{Design Principles}

The soundness of the implementation and the efficiency of its use are due to the following four main principles underlying our design of MOSEL:

1. Definition of a formal semantics for a minimal subset of the logic. Having started with a M2L(Str) implementation for finite strings the semantics is defined in terms of finite-state automata.

2. Layered approach to the logic. We introduce a hierarchy of logic layers, with increasingly powerful constructs, related by either direct embedding or more elaborate encodings as shown in Fig. 1.

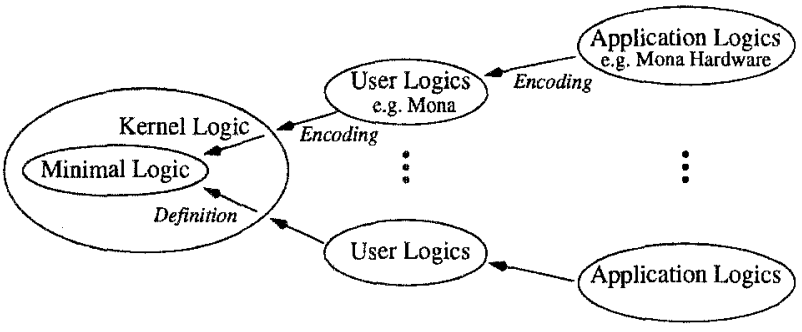

Fig. 1. Layered Logics in MOSEL

The minimal logic contains the minimal set of primitives for which the semantics is formally defined. This set constitutes the reference language for proofs involving semantics, which is very economic e.g. in proofs by structural induction on the constructs of the language. While this is the ideal language for developing the theory, it is not adequate for practical use.

The kernel logic extends the minimal logic by additional (derived) constructs and coincides with the set of constructs actually implemented as primitives in 
the semantic decision procedure. The design of this extension is guided by considerations of efficiency of the computations required in the decision procedure.

A set of generic user logics correspond to an application-independent layer. They extend the kernel logic by derived operators which do not have a direct implementation, but are comfortable for generic applications. User logics may be rather different from the kernel logic and need not be a simple syntactic extension (e.g. the translation of Mona's [5] input syntax into the kernel logic).

A number of application-specific logics, each containing additional admissible predicates and constructs tailored to specific application domains. Here we have direct experience in the domain of verification and synthesis of hardware, where we deal with families of parametric sequential circuits [7].

Due to the principle of implementing outer layers of the logic through successive encodings and definitional extensions to the unique minimal logic, and by making these explicit, the semantic coherence of richer logics with the minimal logic is ensured [6].

Additional advantages of this principle are that the implemented set of primitives is transparent too, and that it is immediately clear which constructs are expensive, due to their (easily computable) definition in terms of kernel logic constructs.

3. Modular design. While other tools, like e.g. Mona, are available only as a single large component, MOSEL is a collection of modules which can be combined or exchanged at need. MOSEL supports flexible adaptation and extension to new input or output formalisms, as well as the interchange of some of its internal components (e.g., users may exchange the BDD package used in the decision procedure, or the automata minimization and determinization algorithms). The aim is that the best-fitting incarnation of the tool may be put together at need, on an application-driven basis, from the collection of existing components.

4. Integratability in a heterogeneous analysis and verification environment. The MOSEL toolset is available within METAFrame ${ }^{\circledR}{ }_{1}^{1}$, a system-level programming environment. It is thus not just a mere stand-alone tool, but its decision procedure as well as each single component (compilers, interfacing and visualization components) are also available for use in more complex, even heterogeneous METAFrame synthesized tools.

\section{Implementation}

The current implementation of the MoseL toolset is written in $\mathrm{C} / \mathrm{C}++$ and features three groups of components: decision procedures, translators between different logics, and graphical visualization modules.

Semantic Decision Procedures. The computational kernel constructs for a given formula of the input logic the minimal finite-state automaton characterizing its semantics. The decision procedure is independent to some degree from

\footnotetext{
${ }^{1}$ METAFrame ${ }^{\circledR}$ is a registered trademark.
} 
the chosen input syntax since it works directly on an object-oriented abstract syntax tree.

Logic Translations. A series of interfacing modules translate each of the several logics into the abstract syntax trees accepted by the actual compiler.

Graphical Visualization. Several alternative graphic tools can be used to display the automata generated within MOSEL. As in Mona, it is possible to use the daVinci [3] tool to show the structure of the automata as well as the concrete BDD encoding of the edge labels. Moreover, the bidirectional link of MoseL's automata to METAFrame's ffgraphs library makes it possible to read and generate automata, not only for display but also to be fed into other algorithms and tools of the METAFrame environment.

Automata Representation. The generated automata are deterministic and complete in order to perform an efficient implementation of negation. Since the size of the edge labels' alphabet is exponential in the number of second-order variables, an explicit enumeration of the letters is excluded. Rather, edge labels are represented by Boolean functions characterizing the transitions, which are in turn implemented via BDD techniques [1]. MoseL uses a hybrid representation of the automata: graphs with edges encoded as boolean functions and implemented via BDDs. This is widely sufficient if the complexity of the automata is mainly due to complex edge labels, as in our application, rather than to intricate sequential structure.

Automata Minimization Algorithm. One of the main nontrivial operations on automata is minimization. In MOSEL this is realized via a modification of the well-known Hopcroft algorithm [4], working on sets of labels instead of single labels.

\section{Efficiency}

Already 30 years ago Alonzo Church proposed monadic second-order logic on strings as an appropriate specification formalism for reasoning about sequences of bitvectors [2]. This logic is among the most succinct decidable logics known to capture finite state systems. It is decidable, however, only in non-elementary time: the worst-case complexity is a stack of exponentials of height proportional to the size of the formula, a good reason for it having been considered impractical for a long time. Recently this logic celebrates a certain renaissance: despite the worst-case computational 'intractability' of this logic, relevant practical problems are usually far better behaved and can be solved automatically in reasonable time.

The intrinsic complexity of the logic demands careful implementation. Our implementation reflects the effort for efficiency in two ways. On the one side a kernel logic as an extension of the minimal logic is used. This logic directly supports many precomputed predicates which are frequently used in applications, in order to cut down the computation time. On the other side BDD techniques are used to handle complex edge labels of the finite automata. 


\section{Application Domains}

The monadic second-order logic over finite strings conveniently combines two important features in a single formalism: It is both an abstract specification language and an effective programming language.

Fields of application have been the specification, verification, and synthesis, in a fully automatic manner, of relevant classes of parametric systems. In particular, the logic can be used profitably as a description language for model-based analysis of software as well as of hardware systems [7] and is therefore a good candidate formalism for hardware/software codesign. Some examples of distributed systems have been addressed too [5]. Every specification can be translated into an equivalent finite-state automaton, and thus is decidable and executable.

\section{Status and Future Work}

At the moment we have implemented in MOSEL a semantic decision procedure for monadic second-order logic over finite strings and a set of interface modules. Decision procedures for other variants of the logic as well as further support components are planned to follow at need. Nevertheless, the design principles and the overall system concept of MOSEL are valid in general.

Future work will follow three main threads. As an alternative to the Mona user logic we plan to implement a typed predicate logic with facilities for userspecific extensions. At the application layer we are working on the embedding of standard HDL languages, like BLIFF. Finally, future extensions of MOSEL will support other semantic theories e.g. like finite trees or finite sets.

\section{References}

1. R.E. Bryant: "Graph-based algorithms for boolean function manipulation," IEEE Trans. Computing, vol. C-35(8), August 1986, pp. 677-691.

2. A. Church: "Logic, arithmetic and automata," Proc. Int. Congr. Math., Almqvist and Wiksells, Uppsala 1963, pp. 23-35.

3. daVinci: the tool is available via ftp at site ftp://ftp.uni-bremen.de/pub/graphics/daVinci

4. J. Hopcroft: "An $n \log n$ algorithm for minimizing states in a finite automaton," Proc. Int. Symp. on Theory of Machines and Computations, Technion, Haifa (IL), Aug. 1971, pp.189-196.

5. J. Henriksen, J. Jensen, M. Jørgensen N. Klarlund, R. Paige, T. Rauhe, A. Sandholm: "Mona: Monadic second-order logic in practice," Proc. of TACAS'95, Aarhus (DK), May 1995, LNCS 1019, Springer Verlag, pp. 89-110.

6. P. Kelb, T. Margaria, M. Mendler, C. Gsottberger: "Mosel: A Flexible Toolset for Monadic Second-Order Logic," TACAS'97, Enschede (NL), April 1997, LNCS 1217, Springer Verlag, pp. 183-202.

7. T. Margaria: "Fully Automatic Verification and Error Detection for Parameterized Iterative Sequential Circuits", Proc. TACAS'96, Passau (D), March 1996, LNCS 1055, Springer Verlag, pp. 258-277. 